\title{
25 Research Square \\ Notch1 Signaling Modulates Hypoxia-induced Multidrug Resistance of Human Laryngeal Cancer Cells
}

\section{Dawei Li}

Shanghai Jiaotong University School of Medicine Xinhua Hospital

\section{Dan Xu}

Tongji University Affilliated Yangpu Hospital: Shanghai Yangpu District Central Hospital

\section{Penghui Chen}

Shanghai Jiaotong University School of Medicine Xinhua Hospital

Jin Xie ( $\nabla$ xiejin@xinhuamed.com.cn)

Shanghai Jiaotong University School of Medicine Xinhua Hospital https://orcid.org/0000-0002-17614397

\section{Research Article}

Keywords: Laryngeal neoplasms, Cell hypoxia, Notch1, Multi-drug resistance

Posted Date: September 14th, 2021

DOl: https://doi.org/10.21203/rs.3.rs-869230/v1

License: (c) (i) This work is licensed under a Creative Commons Attribution 4.0 International License. Read Full License 


\section{Abstract}

Background: Laryngeal carcinoma is one of the common malignant tumors of the head and neck. Multidrug resistance (MDR) remains a critical problem in the chemotherapy for patients with laryngeal cancer. This study aims to clarify the role and mechanisms of Notch1 signaling on MDR induced by hypoxia in laryngeal cancer cells.

Methods and Results: Laryngeal carcinoma cells were cultured under normoxia or hypoxia. Notch1 expression was inhibited by small interfering RNA (siRNA). The expression of Notch1, Hes1, Hey 1, MDR1 and survivin mRNA was determined by Real-time PCR. The expression of Notch1, Notch1 intracellular domain (N1ICD), MDR1/P-gp and survivin protein was detected by Western blot. Current research showed that hypoxia could upregulate Notch1 expression and the activity of Notch1 signaling. Furthermore, suppression of Notch1 expression could effectively down-regulate the activity of Notch1 signaling and the expression of MDR and survivin genes in laryngeal cancer cells under hypoxia $(P<0.05)$. Cell Counting Kit-8 (CCK-8) assay confirmed that the sensitivity of hypoxic laryngeal cancer cells to a variety of drugs could be up-regulated by suppressing Notch1 expression $(P<0.05)$. Additionally, flow cytometry (FCM) showed that suppression of Notch1 expression significantly increased cisplatin-induced apoptosis and intracellular Rh123 (Rh123) accumulation in hypoxic laryngeal carcinoma cells $(P<0.05)$.

Conclusions: Notch1 signalling could be regarded as a pivotal regulator for mediating hypoxia-induced MDR in laryngeal cancer cells by regulating survivin-mediated apoptosis resistance and MDR1/P-gpmediated drug transport.

\section{Introduction}

Laryngeal carcinoma is one of the common malignant tumors of the head and neck. As we know, concurrent chemoradiation has been considered as the primary treatment for locally advanced laryngeal cancer. However, MDR remains a critical problem in the chemotherapy for patients with laryngeal cancer. Unfortunately, the regulatory mechanisms related to MDR of laryngeal carcinoma still remain unclear.

Hypoxia could be served as an essential character of the microenvironment within human solid tumors. It is well-known that hypoxia can cause a series of functional adaptive responses of tumor cells, includig MDR [1-3], which is mediated by a variety of mechanisms. Previously, our in vitro study has confirmed that hypoxia could significantly induce MDR of laryngeal cancer cells [4]. To our knowledge, the molecular mechanisms of hypoxia-induced MDR in laryngeal cancer cells are not fully elucidated.

Notch signaling is regarded as a highly conserved intercellular signaling pathway for the regulation of various biological behaviors in tumor cells under hypoxic microenvironment, which are achieved by regulating the expression of downstream target genes [5, 6]. So far, a series of documents have already demonstrated that aberrant expression of Notch receptors or ligands can be observed in a variety of malignancies, which might be involved in malignant progression [7-9]. Almostly consistent with the study 
of Meng-Yuan Dai et al [10], our previous study has found that Notch1 expression in laryngeal cancer tissues was evidently higher than that of laryngeal normal tissues, and was related to lymph node metastasis and clinical stage [11], suggesting that Notch signaling might play a pivotal role in regulation of malignant progression of laryngeal cancer. Recently, a number of studies have confirmed that Notch1 signaling is involved in regulating MDR of various neoplasic cells [12-14]. Furthermore, several studies have indicated that Notch1 expression has a positive correlation with cisplatin $[15,16]$ and paclitaxel [16] resistance in head and neck squamous cell carcinoma. The above studies suggest that Notch1 signaling may be involved in regulating MDR of laryngeal cancer cells in the hypoxic microenvironment. Up to now, there is no relevant literature report.

In the current study, we were to investigate the regulatory role of Notch1 signaling in hypoxia-induced MDR of laryngeal cancer cells and clarify its possible molecular mechanisms.

\section{Materials And Methods}

Cell lines and cell culture

Laryngeal carcinoma cell lines Hep-2 and AMC-HN-8 were gained from the Institute of Biochemistry and Cell Biology, Shanghai Institute for Biological Sciences, Chinese Academy of Sciences. Neoplasic cells were cultured in DMEM (Gibco Corporation, USA) which was supplemented with 1\% penicillin / streptomycin (Invitrogen) and 10\% fetal bovine serum (Hyclone, USA). For normoxic conditions, cells were placed in an incubator at $37^{\circ} \mathrm{C}$ in an atmosphere of $21 \% \mathrm{O}_{2}, 74 \% \mathrm{~N}_{2}$ and $5 \% \mathrm{CO}_{2}$. For hypoxic conditions, cells were placed in a hypoxic incubator (NuaireTM US autoflow $\mathrm{CO}_{2}$ water jacketed incubator) at $37^{\circ} \mathrm{C}$ containing $1 \% \mathrm{O}_{2}, 94 \% \mathrm{~N}_{2}$ and $5 \% \mathrm{CO}_{2}$.

Cell transfection

The double-stranded siRNA oligonucleotide targeting human Notch1 gene (Notch1-siRNA) (sense: 5'-CAGGGAGCAUGUGUAACAUTT-3', anti-sense: 5'-AUGUUACACAUGCUCCCUGTT-3') and the scrambled siRNA (sense: 5'-UUCUCCGAACGUGUCACGUTT-3', antisense: 5'-ACGUGACACGUUCGGAGAATT-3') were both synthesized by Shanghai Genepharma Co. Ltd. (China). After 24 hours of culture in antibiotic-free medium, laryngeal cancer cells were transfected with siRNA (100 nM) using Lipofectamine 2000. Then, cells should be collected for further examine after transfection for 24 hours.

Real-time PCR analysis

Trizol reagent (Invitrogen) extracted total RNA from neoplastic cells. According to reverse transcription kit instructions, cDNA synthesis was implemented. The primers for PCR were as follows: Notch 1 forward, 5'-CTACCTGTCAGACGTGGCCT-3' and reverse, 5'-CGCAGAGGGTTGTATTGGTT-3'. Hes 1 forward, 5'-TCTGAGCCAGCTGAAAACAC-3' and reverse, 5'-GGTACTTCCCCAGCACACTT-3'. Hey 1 forward, 5'-GGCTCCTTCCACTTACTGTCTC-3' and reverse, 5'- ACTTTCCCCTCCCTCATTCTAC-3'. MDR 1 forward, 5'-CTTCAGGGTTTCACATTTGGC-3' and reverse, 5'- GGTAGTCAATGCTCCAGTGG-3'. Survivin forward, 
5'-CTTCATCCACTGCCCCAC-3' and reverse, 5'- ACTTTCTCCGCAGTTTCCTC-3'. GAPDH (internal control) forward, 5'-CATCTTCCAGGAGCGAGA-3' and reverse, 5'-TGTTGTCATACTTCTCAT-3'. As conducted in our previous study [4], Real-time PCR quantified the expression of Notch1, Hes1, Hey1, MDR1, survivin and GAPDH mRNA using SYBR Green PCR kit (Takara Biotechnology Co., Ltd., Dalian, China). Real-time PCR results were analyzed by the $2^{-\triangle \triangle C T}$ method [17].

Western blot analysis

Laryngeal cancer cells were collected and lysed with RIPA lysis buffer for half an hour. Equal amounts of lysate proteins $(25 \mu \mathrm{g})$ went electrophoresis in SDS-PAGE (5\% stacking gel and $8 \%$ separating gel) and transferred to a PVDF membrane (Millipore), blocked with 5\% skim milk solution for 2 hours at room temperature. Then, the membranes were incubated with primary antibodies (Notch1 1:1000, rabbit antihuman; N1ICD 1:1000, rabbit anti-human; Survivin 1:1000, rabbit anti-human; MDR1/P-gp 1:200, mouse anti-human; GAPDH, 1:1000, mouse anti-human) overnight at $4^{\circ} \mathrm{C}$, and the secondary antibodies (1: 5000; room temperature, 1 hour). Finally, the immunoreactive proteins were visualized by electrogenerated chemiluminescence.

Cell cytotoxicity assay

CCK-8 assay was to assess the sensitivity of neoplastic cells to adriamycin, paclitaxel, cisplatin, 5-FU and gemcitabine. The cells were placed in 96 -well culture panels $\left(5 \times 10^{3}\right.$ cells/well). After 12 hours, cells were dealed with a certain dose of chemotherapeutic drugs and cultured for another 48 hours under hypoxia or normoxia. As mentioned in previous study [4], the drug concentration $\left(\mathrm{IC}_{50}\right)$ which lead to a $50 \%$ reduction in cell number could be calculated.

Rhodamine 123 accumulation assay

FCM assay was used to analyze the accumulation of Rh123 in Hep-2 and AMC-HN-8 cells as described previously [18]. The FACSCalibur flow cytometer (BD Biosciences, Franklin Lakes, NJ, USA) evaluated the cell suspension by using $488 \mathrm{~nm}$ excitation. Then, Cell-Quest ${ }^{\mathrm{TM}}$ software (BD Biosciences) analyzed the experimental data.

Cell apoptosis analysis

Hep-2 (3×105 cells/well) and AMC-HN-8 (4×105 cells/well) cells were plated in six-well plates and cultured overnight at $37^{\circ} \mathrm{C}$. Then, cells were cultured in hypoxia or normoxia for 12 hours after culture medium was renewed. Next, cell culture further lasted 48 hours after adding cisplatin to each well until the concentration reached $2.5 \times 10-9 \mathrm{M}$. As our previous research, the apoptosis index (Al) of cells was assessed by FCM and Annexin-V-FITC/propidium iodide (PI) staining method [4]. Finally, cell apoptosis rate was measured at the average fluorescence intensity.

Statistical analysis 
The comparison of quantitative variables was assessed by Student's t-test analysis with SPSS20.0. That values of $P$ less than 0.05 was regarded as statistically significant.

\section{Results}

Hypoxia up-regulated Notch1 expression and the activity of Notch1 signaling in laryngeal carcinoma cells

Laryngeal cancer cells were cultured under normoxic or hypoxic conditions for $12,24,48$ hours. Real-time PCR assay determined that hypoxia could obviously induce the expression of Notch1, Hes1, Hey1 mRNA in neoplastic cells $(P<0.05)$ (Fig. 1A-C). Hes1 and Hey1 belong to the downstream target genes of Notch signaling, and are usually used to reflect the activity of Notch signaling. Similarly, Western blot assay showed that Notch1 and N1ICD expression in laryngeal cancer cells was up-regulated with exposure to hypoxia $(P<0.05)$ (Fig. 1D, E). N1ICD could be considered as the active ingredient of Notch1 protein. Thus, the above data indicated that hypoxia could up-regulate Notch1 expression and the activity of Notch1 signaling.

Suppression of Notch 1 expression down-regulated the activity of Notch1 signaling in hypoxic laryngeal carcinoma cells

Real-time PCR exhibited that the expression of Notch1, Hes1 and Hey1 mRNA in Notch1-siRNA group was evidently less than that of control groups $(P<0.05)$ (Fig. 2A-C). Meanwhile, Western blot assay revealed that Notch1 and N1ICD protein expression in Notch1-siRNA group was less than that of control groups $(P<0.05)$ (Fig. 2D, E). The above data demonstrated that suppression of Notch 1 expression could down-regulate the activity of Notch1 signaling in hypoxic laryngeal cancer cells.

Suppression of Notch1 expression inhibited multidrug resistance of laryngeal carcinoma cells under hypoxia

Our study compared the drug sensitivity of Notch1-siRNA group with that of control groups by CCK-8 method. As can be seen in Table 1 and 2, the results showed that the sensitivity of hypoxic Hep-2 and AMC-HN-8 cells to a variety of drugs was obviously enhanced by inhibiting Notch 1 expression $(P<0.05)$.

Suppression of Notch1 expression inhibited the expression of MDR1 and survivin genes in hypoxic laryngeal cancer cells

Real-time PCR assay exhibited that MDR1 and survivin mRNA expression in Notch1-siRNA group was obviously less than that of control groups $(P<0.05)$ (Fig. 3A-C). Besides, Western blot assay showed that MDR1/P-gp and survivin protein expression in Notch1-siRNA group was also less than that of control groups $(P<0.05)$ (Fig. 3D, E). The above data indicated that MDR1 and survivin expression in hypoxic laryngeal cancer cells was down-regulated by inhibiting Notch1 expression.

Suppression of Notch 1 expression increased drug accumulation in hypoxic laryngeal cancer cells 
FCM assay showed that the positive percentage of Rh123 in Notch1-siRNA group of Hep-2 cells was evidently higher than that of control groups $(89.48 \pm 1.97 \%$ vs. $70.39 \pm 1.66 \%, 70.63 \pm 0.71 \% ; P<0.05)$ (Fig. 4A); Besides, the positive percentage of Rh123 in Notch1-siRNA group of AMC-HN-8 cells was also higher than that of control groups $(92.35 \pm 2.13 \%$ vs. $73.12 \pm 3.10 \%, 72.84 \pm 2.24 \% ; P<0.05)$ (Fig. 4B). The above data revealed that suppression of Notch1 expression could enhance drug accumulation in hypoxic laryngeal cancer cells.

Suppression of Notch1 expression enhanced cisplatin-induced apoptosis of hypoxic laryngeal cancer cells

Annexin-V/PI staining method showed that Al of Hep-2 cells in Notch1-siRNA group was obviously higher than that of control groups ( $46.69 \pm 0.68 \%$ vs. $20.56 \pm 0.85 \%, 20.40 \pm 0.58 \% ; P<0.05)$ (Fig. 4 C); Likewise, Al of AMC-HN-8 cells in Notch1-siRNA group was also higher than that of control groups $(51.67 \pm 0.69 \%$ vs. $31.27 \pm 0.54 \%, 31.19 \pm 0.57 \% ; P<0.05$ ) (Fig. 4D). The above data demonstrated that suppression of Notch1 expression enhanced cisplatin-induced apoptosis of hypoxic laryngeal cancer cells.

\section{Discussion}

Notch signaling is a crucial signal transduction pathway for the regulation of biological behaviors of neoplasic cells under hypoxia [5]. Previously, the data of Meng-Yuan Dai et al. [10] and our work [11] have demonstrated that high expression of Notch1 in laryngeal cancer tissues was associated with lymph node metastasis. Furthermore, current research exhibited that hypoxia could enhance Notch1 expression and the activity of Notch 1 signaling in laryngeal cancer cells. The above results suggested that, in the hypoxic microenvironment of laryngeal cancer tissue, Notch1 signaling might take an important part in regulation of malignant phenotypes.

Up to date, a number of studies in other oncology fields have shown that Notch1 signaling is involved in regulating MDR of various neoplastic cells [12-14]. Furthermore, the studies of Zuping Zhang et al. [16] and Feng Gu et al. [15] demonstrated that Notch1 expression was positively correlated with chemotherapy resistance of head and neck carcinoma. And then, the present work showed that the sensitivity of hypoxic laryngeal cancer cells to a variety of chemotherapy drugs was obviously enhanced by restraining the activity of Notch1 signaling. That is to say, Notch1 signaling might take a significant part in mediating hypoxia-induced MDR in laryngeal cancer cells.

MDR1/P-gp, as a critical drug transporter, affects on the regulating of intracellular drug concentrations. MDR1/P-gp has been confirmed as an important regulator of MDR in laryngeal cancer cells [19, 20]. Furthermore, our previous work has suggested that MDR1/P-gp could serve a significant role in regulating hypoxia-induced MDR in laryngeal carcinoma cells through cellular drug effluxing mechanism [21]. Recently, Jiayuan Huang et al. [22] have indicated that Notch-1 signaling may play a role in regulating chemoresistance in lung adenocarcinoma by mediating MDR1 expression. Likewise, our present work has elucidated that suppression of Notch1 expression could down-regulate MDR1 expression in hypoxic laryngeal carcinoma cells, and reduce the drug efflux ability of neoplastic cells. 
Consequently, it is suggested that Notch1 signaling might participate in the regulation of MDR1/P-gpmediated drug transport in laryngeal cancer cells under hypoxia.

Survivin belongs to the inhibitor of apoptosis family and participates in the apoptosis regulation of laryngeal cancer cells [23,24]. Besides, the study of Himani Sharma et al. [25] has indicated that survivin takes part in the regulation of drug sensitivity of head and neck squamous cell carcinoma cells, including Hep-2 cells. Recently, our research has already confirmed that survivin might play a regulatory role in hypoxia-induced MDR of laryngeal carcinoma cells by regulating apoptosis resistance [26]. Moreover, several studies have identified that Notch-1 signaling might regulate survivin expression in basal breast cancer cells [27] and lung cancer cells [28]. In this series, our work has confirmed that suppression of Notch1 expression could down-regulate survivin expression in hypoxic laryngeal carcinoma cells, and enhance cisplatin-induced apoptosis of neoplastic cells. Accordingly, it is indicated that Notch1 signaling might be involved in the regulation of survivin-mediated apoptosis resistance of laryngeal cancer cells under hypoxia.

In summary, current research indicates that Notch1 signaling may play an important role in regulating hypoxia-induced MDR in laryngeal cancer cells by regulating survivin-mediated apoptosis resistance and MDR1/P-gp-mediated drug transport. Further study is needed to determine the role and mechanisms of Notch1 signaling in hypoxia-induced MDR of laryngeal carcinoma cells through in vivo experiments.

\section{Declarations}

Acknowledgments

We are very grateful to Prof. Xue-mei Tong, Department of Biochemistry and molecular Biology, Shanghai Jiao Tong University School of Medicine, for technical support in the research process. This work was supported by grants from the National Natural Science Foundation of China (No. 81502339, 81402325, 81874151), Grant 20184 Y0241 from Shanghai Municipal Population and Family Planning Commission, Grant 202040162 from Shanghai Municipal Health Bureau.

Funding This work was supported by grants from the National Natural Science Foundation of China (No. 81502339, 81402325, 81874151), Grant 20184Y0241 from Shanghai Municipal Commission of Health and Family Planning, Grant 202040162 from Shanghai Municipal Health Commission.

Conflicts of interest/Competing interests The author reports no conflicts of interest/Competing interests in this work.

Availability of data and material Available.

Code availability Not applicable.

Authors' contributions Dawei Li, study execution, data acquisition, analysis and interpretation, manuscript drafting and revising, final approval, and accountability for all aspects of the work; Dan Xu, 
study execution, data acquisition, analysis and interpretation, manuscript drafting and revising, final approval, and accountability for all aspects of the work; Penghui Chen, study execution, data acquisition, analysis and interpretation; Jin Xie, study design, data analysis and interpretation, manuscript revising, final approval, and accountability for all aspects of the work.

Ethics approval Not applicable.

Consent to participate Not applicable.

Consent for publication All authors approved to publish this article.

\section{References}

1. Xiaochun Shen, Qiaoming Zhi, Yunliang Wang et al (2017) Hypoxia Induces Multidrug Resistance via Enhancement of Epidermal Growth Factor-Like Domain 7 Expression in Non-Small Lung Cancer Cells. Chemotherapy 62:172-180

2. Hong Zhu, Shun-feng Luo, Ji Wang et al (2012) Effect of environmental factors on chemoresistance of HepG2 cells by regulating hypoxia-inducible factor-1a. Chin Med J (Engl) 125:1095-1103

3. H Zhu, X P Chen, S F Luo et al (2005) Involvement of hypoxia-inducible factor-1-alpha in multidrug resistance induced by hypoxia in HepG2 cells. J Exp Clin Cancer Res 24:565-574

4. Da-Wei Li, Pin Dong, Fei Wang et al (2013) Hypoxia induced multidrug resistance of laryngeal cancer cells via hypoxia-inducible factor-1a. Asian Pac J Cancer Prev 14:4853-4858

5. Cecilia Sahlgren, Maria V Gustafsson, Shaobo Jin et al (2008) Notch signaling mediates hypoxiainduced tumor cell migration and invasion. Proc Natl Acad Sci U S A 105:6392-6397

6. Giovanna Danza, Claudia Di Serio, Fabiana Rosati et al (2012) Notch signaling modulates hypoxiainduced neuroendocrine differentiation of human prostate cancer cells. Mol Cancer Res 10:230-238

7. Binyu Zhao, Shanshan Hu, Qingqing Xiao et al (2020) Expression of NOTCH receptors and ligands and prognosis of hepatocellular carcinoma. Biomark Med 14:1631-1639

8. Jen-Tsun Lin, Mu-Kuan Chen, Kun-Tu Yeh et al (2010) Association of high levels of Jagged-1 and Notch-1 expression with poor prognosis in head and neck cancer. Ann Surg Oncol 17:2976-2983

9. Jianpeng Wang, Chao Wang, Qinghai Meng et al (2012) siRNA targeting Notch-1 decreases glioma stem cell proliferation and tumor growth. Mol Biol Rep 39:2497-2503

10. Meng-Yuan Dai, Fang Fang, You Zou et al (2015) Downregulation of Notch1 induces apoptosis and inhibits cell proliferation and metastasis in laryngeal squamous cell carcinoma. Oncol Rep 34:31113119

11. Dawei Li, Pin Dong, Chunping Wu et al (2014) Notch1 overexpression associates with poor prognosis in human laryngeal squamous cell carcinoma. Ann Otol Rhinol Laryngol 123:705-710

12. Xingwang Kuai, Lizhou Jia, Tingting Yang et al (2020) Trop2 Promotes Multidrug Resistance by Regulating Notch1 Signaling Pathway in Gastric Cancer Cells. Med Sci Monit 26:e919566 
13. C Li, D Guo, B Tang et al (2016) Notch1 is associated with the multidrug resistance of hypoxic osteosarcoma by regulating MRP1 gene expression. Neoplasma 63:734-742

14. Sungpil Cho, Meiling Lu, Xiaolong He et al (2011) Notch1 regulates the expression of the multidrug resistance gene ABCC1/MRP1 in cultured cancer cells. Proc Natl Acad Sci U S A 108:20778-20783

15. Feng Gu, Yongjie Ma, Zuping Zhang et al (2010) Expression of Stat3 and Notch1 is associated with cisplatin resistance in head and neck squamous cell carcinoma. Oncol Rep 23:671-676

16. Zuping Zhang, Zhongxin Zhou, Mingde Zhang et al (2019) High Notch1 expression affects chemosensitivity of head and neck squamous cell carcinoma to paclitaxel and cisplatin treatment. Biomed Pharmacother 118:109306.

17. K J Livak, T D Schmittgen (2001) Analysis of relative gene expression data using real-time quantitative PCR and the 2(-Delta Delta C(T)) Method. Methods 25:402-408

18. C Ludescher, J Thaler, D Drach et al (1992) Detection of activity of P-glycoprotein in human tumour samples using rhodamine 123. $\mathrm{Br} \mathrm{J}$ Haematol 82:161-168

19. Li Li, Alice C Jiang, Pin Dong et al (2007) The characteristics of Hep-2 cell with multiple drug resistance induced by Taxol. Otolaryngol Head Neck Surg 137:659-664

20. Huang Zhigang, Zhong Qi, Fang Jugao et al (2009) Reverse multidrug resistance in laryngeal cancer cells by knockdown MDR1 gene expression. J Otolaryngol Head Neck Surg 38:440-448

21. Dawei Li, Liang Zhou, Jiameng Huang et al (2016) Effect of multidrug resistance 1/P-glycoprotein on the hypoxia-induced multidrug resistance of human laryngeal cancer cells. Oncol Lett 12:1569-1574

22. Jiayuan Huang, Yitian Chen, Junyang Li et al (2016) Notch-1 Confers Chemoresistance in Lung Adenocarcinoma to Taxanes through AP-1/microRNA-451 Mediated Regulation of MDR-1. Mol Ther Nucleic Acids 5:e375

23. Xiu-mei Chen, Xin-yong Luan, Da-peng Lei et al (2008) Suppression of survivin expression by short hairpin RNA induces apoptosis in human laryngeal carcinoma cells. ORL J Otorhinolaryngol Relat Spec 70:168-175

24. Da-Wei Li, Shang Gao, Bin Shen et al (2011) Effect of apoptotic and proliferative indices, Pglycoprotein and survivin expression on prognosis in laryngeal squamous cell carcinoma. Med Oncol 28 Suppl 1:S333-S340

25. Himani Sharma, Sudip Sen, Lorenzo Lo Muzio et al (2005) Antisense-mediated downregulation of anti-apoptotic proteins induces apoptosis and sensitizes head and neck squamous cell carcinoma cells to chemotherapy. Cancer Biol Ther 4:720-727

26. Dan Xu, Da Wei Li, Jin Xie et al (2019) Effect and Mechanism of Survivin on Hypoxia-Induced Multidrug Resistance of Human Laryngeal Carcinoma Cells. Biomed Res Int. https://doi.org/10.1155/2019/5696801

27. Connie W Lee, Karl Simin, Qin Liu et al (2008) A functional Notch-survivin gene signature in basal breast cancer. Breast Cancer Res 10:R97 
28. Yuqing Chen, Dianming Li, Hongli Liu et al (2011) Notch-1 signaling facilitates survivin expression in human non-small cell lung cancer cells. Cancer Biol Ther 11:14-21

\section{Tables}

Table 1 Effect of Notch1 silencing on chemosensitivity in hypoxic Hep-2 cells

\begin{tabular}{|llll|}
\hline Drug & $\mathrm{IC}_{50}(\mu \mathrm{g} / \mathrm{ml})$ & & \\
& Blank control & Negative control & Notch1-siRNA \\
\hline Paclitaxel & $37.71 \times 10-3 \pm 0.18$ & $38.52 \times 10-3 \pm 0.29$ & $4.34 \times 10-3 \pm 0.13^{*}$ \\
\hline $5-\mathrm{Fu}$ & $243.04 \pm 0.84$ & $241.24 \pm 0.88$ & $26.17 \pm 0.27^{*}$ \\
\hline Doxorubicin & $3.91 \pm 0.07$ & $3.78 \pm 0.11$ & $1.08 \pm 0.21^{*}$ \\
\hline Gemcitabine & $38.26 \pm 0.29$ & $39.12 \pm 0.53$ & $11.46 \pm 0.12^{\star}$ \\
\hline Cisplatin & $8.91 \pm 0.11$ & $8.73 \pm 0.21$ & $2.03 \pm 0.18^{*}$ \\
\hline
\end{tabular}

$\mathrm{IC}_{50}$ is the concentration of each drug that caused a $50 \%$ reduction in the number of cells.

Mean \pm SD of three individual experiments are shown. *: P凶0.05 vs. Blank control and Negative control.

Table 2 Effect of Notch1 silencing on chemosensitivity in hypoxic AMC-HN-8 cells

\begin{tabular}{|llll|}
\hline Drug & $\mathrm{IC}_{50}(\mu \mathrm{g} / \mathrm{ml})$ & & \\
& Blank control & Negative control & Notch1-siRNA \\
\hline Paclitaxel & $35.18 \times 10-3 \pm 0.78$ & $36.12 \times 10-3 \pm 0.21$ & $3.06 \times 10-3 \pm 0.31^{*}$ \\
\hline $5-\mathrm{Fu}$ & $227.07 \pm 1.24$ & $228.28 \pm 1.37$ & $23.61 \pm 0.32^{*}$ \\
\hline Doxorubicin & $3.68 \pm 0.35$ & $3.83 \pm 0.41$ & $0.81 \pm 0.42^{*}$ \\
\hline Gemcitabine & $35.31 \pm 0.25$ & $36.25 \pm 0.48$ & $9.36 \pm 0.28^{*}$ \\
\hline Cisplatin & $8.61 \pm 0.17$ & $8.55 \pm 0.31$ & $1.56 \pm 0.34^{*}$ \\
\hline
\end{tabular}

$\mathrm{IC}_{50}$ is the concentration of each drug that caused a $50 \%$ reduction in the number of cells.

Mean \pm SD of three individual experiments are shown. *: Pه0.05 vs. Blank control and Negative control.

\section{Figures}




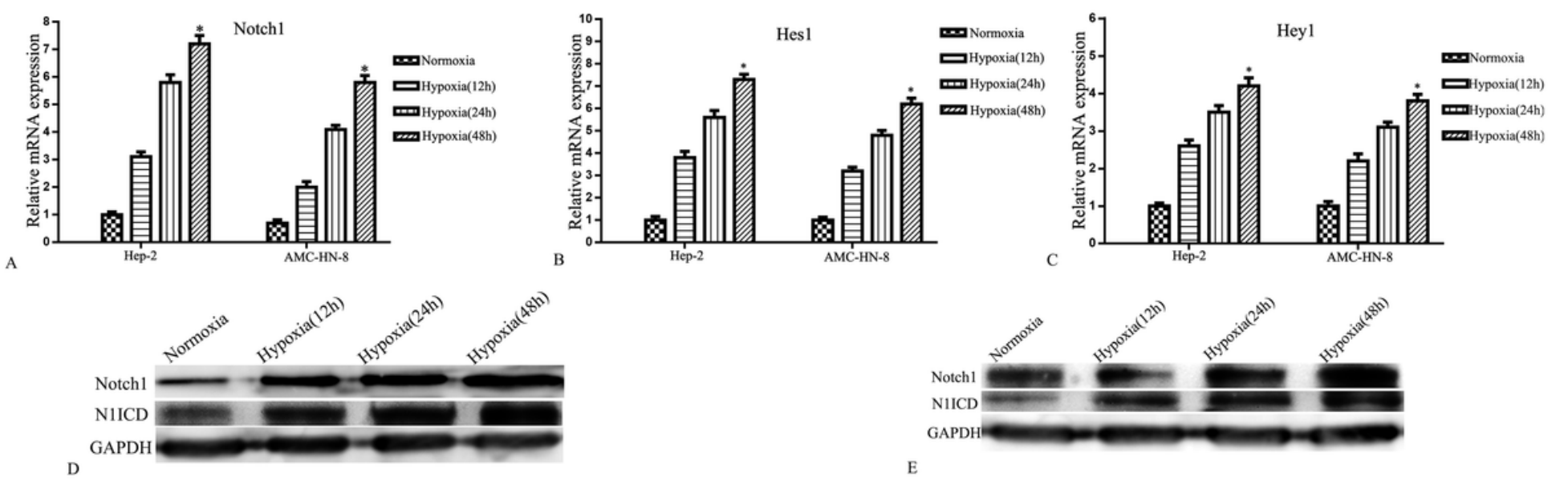

Figure 1

Changes in Notch1 expression and the activity of Notch1 signaling in laryngeal carcinoma cells under normoxia or hypoxia. Real-time PCR estimated the expression of Notch1 (A), Hes1 (B) and Hey1 (C) mRNA in neoplastic cells. Western blot evaluated the expression of Notch1 and N1ICD protein in Hep-2 (D) and AMC-HN-8 (E) cells. ${ }^{*} \mathrm{P}<0.05$, versus control groups
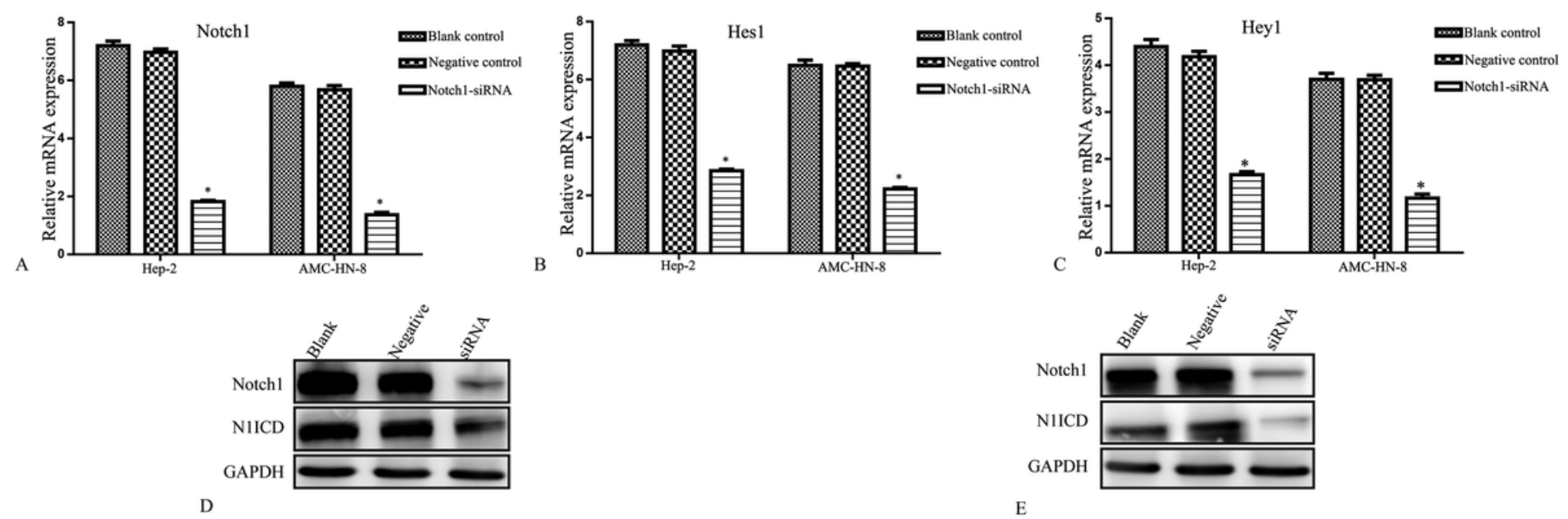

Figure 2

Effects of Notch1-siRNA on the activity of Notch1 signaling in hypoxic laryngeal carcinoma cells. Neoplastic cells were transfected with the scrambled siRNA or Notch1-siRNA. Real-time PCR assessed the expression of Notch1 (A), Hes1 (B) and Hey1 (C) mRNA in Hep-2 and AMC-HN-8 cells under hypoxia. Western blot estimated the expression of Notch1 and N1ICD protein in Hep-2 (D) and AMC-HN-8 (E) cells under hypoxia. ${ }^{*} \mathrm{P}<0.05$, versus control groups 

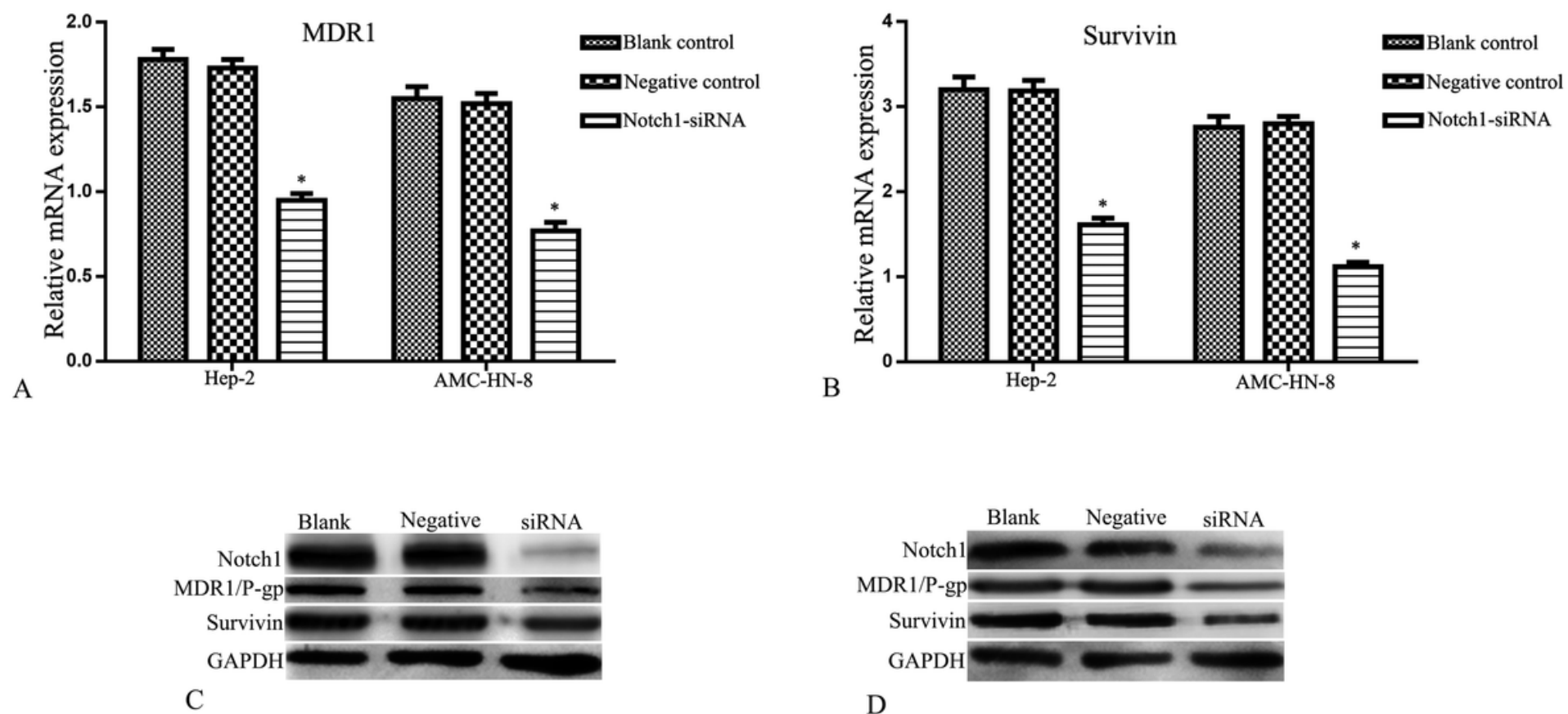

Figure 3

Effects of Notch1-siRNA on MDR1 and survivin expression in hypoxic laryngeal cancer cells. The cells were transfected with the scrambled siRNA or Notch1-siRNA. Real-time PCR assessed the expression of MDR1 (A) and survivin (B) mRNA in neoplastic cells under hypoxia. Western blot estimated the expression of MDR1/P-gp and survivin protein in Hep-2 (C) and AMC-HN-8 (D) cells under hypoxia. ${ }^{*} \mathrm{P}<0.05$, versus control groups 

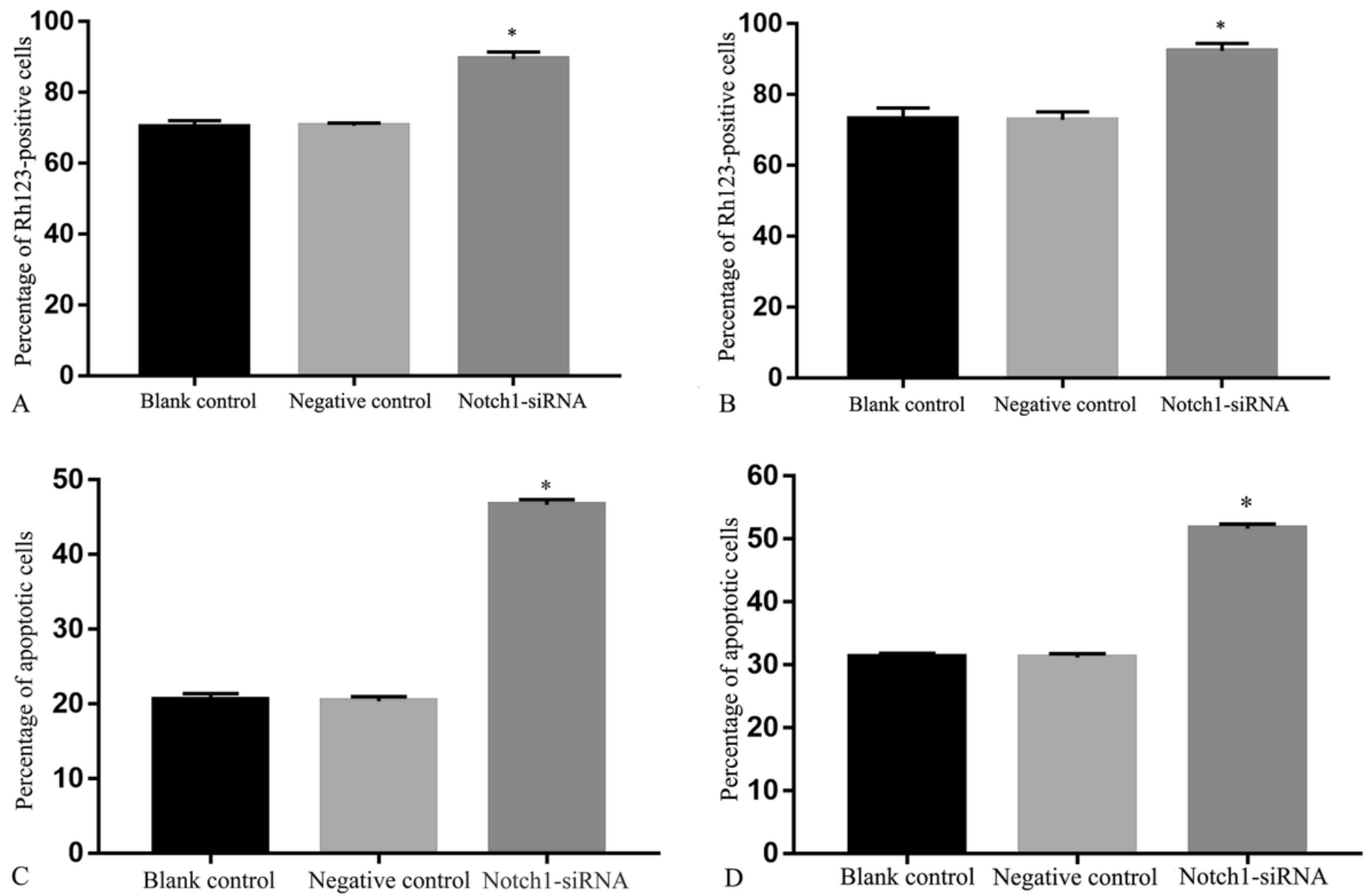

Figure 4

Effects of Notch1-siRNA on drug efflux capacity and cisplatin-induced apoptosis of hypoxic laryngeal carcinoma cells. FCM assay was used to detect intracellular Rh123 accumulation and apoptosis rate of neoplastic cells. Suppression of Notch1 expression could increase the positive percentage of Rh123 in Hep-2 (A) and AMC-HN-8 (B) cells, and enhance the apoptosis rate of Hep-2 (C) and AMC-HN-8 (D) cells induced by cisplatin. ${ }^{*}<<0.05$, versus control groups 